\title{
Determination of thickness and density of a wet multilayer polymer system with sub-nanometer resolution by means of a dual polarization silicon-on-insulator microring
}

\author{
J.-W. Hoste*a,b , Bruno G. De Geest ${ }^{\mathrm{c}}$, Peter Bienstman ${ }^{\mathrm{a}, \mathrm{b}}$ \\ ${ }^{a}$ Photonics Research Group (INTEC), Ghent University - imec, Sint-Pietersnieuwstraat 41, 9000 \\ Ghent, Belgium; ${ }^{\mathrm{b}}$ Center for Nano- and Biophotonics (NB-Photonics), Ghent University, Sint- \\ Pietersnieuwstraat 41, 9000 Ghent, Belgium; ${ }^{\circ}$ Department of Pharmaceutics, Ghent University, \\ Harelbekestraat 72, 9000 Ghent, Belgium.
}

\begin{abstract}
Determination of both thickness and refractive index of a thin biomolecular or polymer layer in wet conditions is a task not easily performed. Available tools such as XPS, AFM, ellipsometry and integrated photonic sensors often have difficulties with the native wet condition of said agents-under-test, perform poorly in the sub-5 $\mathrm{nm}$ regime or do not determine both characteristics in an absolute simultaneous way. The thickness of a multilayer system is often determined by averaging over a large amount of layers, obscuring details of the individual layers. Even more, the interesting behavior of the first bound layers can be covered in noise or assumptions might be made on either thickness or refractive index in order to determine the other. To demonstrate a solution to these problems, a silicon-on-insulator (SOI) microring is used to study the adsorption of a bilayer polymer system on the silicon surface of the ring. To achieve this, the microring is simultaneously excited with TE and TM polarized light and by tracking the shifts of both resonant wavelengths, the refractive index and the thickness of the adsorbed layer can be determined with a resolution on thickness smaller than $0.1 \mathrm{~nm}$ and a resolution on refractive index smaller than 0.01 RIU. An adhesive polyethyleneimine (PEI) layer is adsorbed to the surface, followed by the adsorption of poly(sodium-4-styrene sulfonate) (PSS) and poly(allylamine) hydrochloride (PAH). This high-resolution performance in wet conditions with the added benefits of the SOI microring platform such as low cost and multiplexibility make for a powerful tool to analyze thin layer systems, which is promising to research binding conformation of proteins as well.
\end{abstract}

Keywords: dual polarization, microring resonator, silicon-on-insulator, polymers, sensing, thin layers, integrated photonics, conformation

\section{INTRODUCTION}

In a Nature Chemistry Insight ${ }^{1}$, Connelly points out that a major part in the current development crisis of the pharmaceutical industry lies in our poor understanding of how our drug candidates function at a molecular level. Easy targets no longer exist and as is touched upon by Scannel ${ }^{2}$, a lot of current drugs lack the specificity to reach only the target biomolecule, hitting multiple unintended targets instead and thus causing collateral damage. In order to find these magic bullets we have to figure out what we are aiming at and how these bullets work. As such, there is a need for a high-throughput tool which can monitor different aspects of binding dynamics such as conformation, kinetic response and affinity of binding.

Typical techniques that resolve the conformation of molecules are either bulk techniques or surface techniques. A powerful and accurate bulk technique such as NMR is adequate to study molecular dynamics and conformations ${ }^{3}$, but the signals are notoriously difficult to analyze and it requires a lot of biomolecules. Other bulk techniques such as multiangle light scattering measure the mass and the size of a molecule in an indirect way via the hydrodynamical radius ${ }^{4}$, approximating the shape by a sphere and averaging out over all three dimensions. In general, bulk techniques are not well suited for high-throughput measurements which are crucial for pharmaceutical lead discovery. This is more easily achieved with a surface technique. A large fraction of the existing optical surface techniques are refractive techniques, where the molecules bind to the surface which is probed by an optical mode, sensing the disturbance through a change in its effective index. However, the effective index of the optical mode is sensitive to both changes in the refractive index, 
closely related to the volume density, and the thickness of the bound layer. In essence, the optical mode cannot distinguish between a thin dense film and a thick sparse film. To see if molecules contract or unfold upon a binding event, the physical thickness of the layer needs to be extracted independently. To do so, many of the refractive techniques assume that the refractive index of the layer has a constant value over the time of the experiment. Since the refractive index has a direct relation to the volume density of a particular layer, it is clear that when molecules change their conformation, this refractive index can change just as much as the thickness can. Assuming a certain refractive index would in this case induce errors on the extracted thickness. Other techniques grow a large number of layers and try to fit the measurement results to simulations in order to extract the parameters of a single film 5 , or try to extract both thickness and refractive index independently by using several discrete wavelengths ${ }^{6}$ to probe the film, introducing errors due to dispersion.

The technique we propose to extract both thickness and refractive index simultaneously is a Silicon-on-Insulator (SOI) microring resonator which is excited by a quasi-TE and a quasi-TM mode. Both modes interact differently with the thin molecular layer and via careful data analysis both parameters can be disentangled ${ }^{7}$. Since the optical modes only extend into the exterior of the optical core for about $100 \mathrm{~nm}$, they are especially appropriate to study interface interactions or any events that happen close to the surface. This is usually the region where traditional surface techniques such as ellipsometry and AFM are blind to due to high noise. $\mathrm{In}^{8}$ it is stated that the adsorption of polyelectrolyte layers to the surface has an interesting first region close to the surface which behaves different than a bulk polymer, and consequently difficult to analyze with existing techniques. In this article we showcase the possibility of the sensor by resolving the thickness and refractive index of a thin stack of polyelectrolytes comprising of a polyethyleneimine (PEI) layer, a poly(sodium-4-styrene sulfonate) (PSS) layer and a poly(allylamine) hydrochloride (PAH) layer, in its native wet condition.

\section{DUAL POLARIZATION MICRORING SENSOR}

\subsection{Sensing of the molecular layer}

The microring sensor being presented here is a rectangular wire waveguide in a racetrack resonator configuration. Next to the resonator an access waveguide is located in close proximity to couple the light in and out of the resonator. The optical chip on which several microrings reside, each with its own input and output coupler, is fabricated with $193 \mathrm{~nm}$ deep UV lithography ${ }^{9}$. The $2 \mathrm{D}$ cross section is a silicon rectangle of $220 \mathrm{~nm}$ high and $520 \mathrm{~nm}$ wide, that rests on a silicon dioxide box. These dimensions make sure the waveguide supports a fundamental quasi-TM mode and a fundamental quasi-TE mode. Since these modes are quasi-orthogonal the interaction with the adsorbed polymer layer is quite different. The electric field of the TM mode is not continuous across the top interface of the waveguide, extending the evanescent tail further in to the aqueous cladding than the TE mode. This is illustrated in Figure 1.

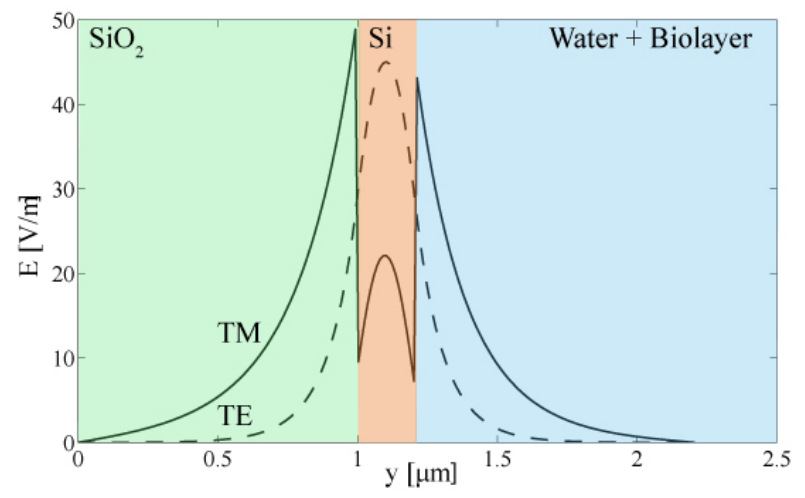

Figure 1. Electric field profile of a quasi-TE and a quasi-TM mode in the horizontal center of the 2D waveguide cross section, as a function of the vertical coordinate. For both polarizations, only the dominant electric field component in the $2 D$ cross section is shown. 
The interaction of the waveguide mode with the thin polyelectrolyte layer is quantified by means of the effective index of this waveguide mode. This effective index is a scalar that acts as a representation of the way the mode conforms to the material system and its geometry. Since the electric field overlaps with the thin polyelectrolyte layer, every change of the composition of this layer is reflected as a change in the effective index. In turn, a perturbation of this effective index will cause a shift of the spectral position of the cavity resonance through the following relation:

$$
\Delta \lambda(n, t)=\frac{\Delta n_{e f f}(n, t) \cdot \lambda}{n_{g}}
$$

Here, $t$ is the thickness of the layer, $n$ is the refractive index of the layer, $n_{\text {eff }}$ is the effective index of the mode, $n_{g}$ is the group index and $\lambda$ is the wavelength of the optical mode. This change in wavelength due to the adsorption of a polyelectrolyte layer can be directly measured by the experimental setup for both TE and TM modes simultaneously. Due to the different field profiles as illustrated in Figure 1, the wavelength shift will also be different for a certain thickness and density of the polyelectrolyte layer and this difference allows us to extract both independent parameters of the layer.

\subsection{Optical design of the sensor}

Designing a ring cavity that supports two modes simultaneously depends heavily on the geometry of the coupling section. We chose to use one coupling section that excites both modes at once. This allows for the light to be routed around the photonics circuit in TE polarization, such that the lossy TM mode only exists in the microring itself. To achieve this, an asymmetrical directional coupler ${ }^{10}$ is used. Due to careful phasematching, this coupling section manages to couple the propagating TE mode in the access waveguide to a TM mode in the ring waveguide. This phasematching is done by choosing a specific combination of widths for the access waveguide and the ring waveguide in the coupling section. This results in a large phase mismatch between the TE mode in the access waveguide and the TE mode in the ring waveguide, rendering coupling almost impossible at conventional gaps. When the gap of the coupling section is small enough however, this phase mismatch can be compensated by substantial spatial overlap between the electric fields of the TE mode of the access waveguide and the TE mode of the ring waveguide. These devices were designed using the IPKISS parametric design framework.
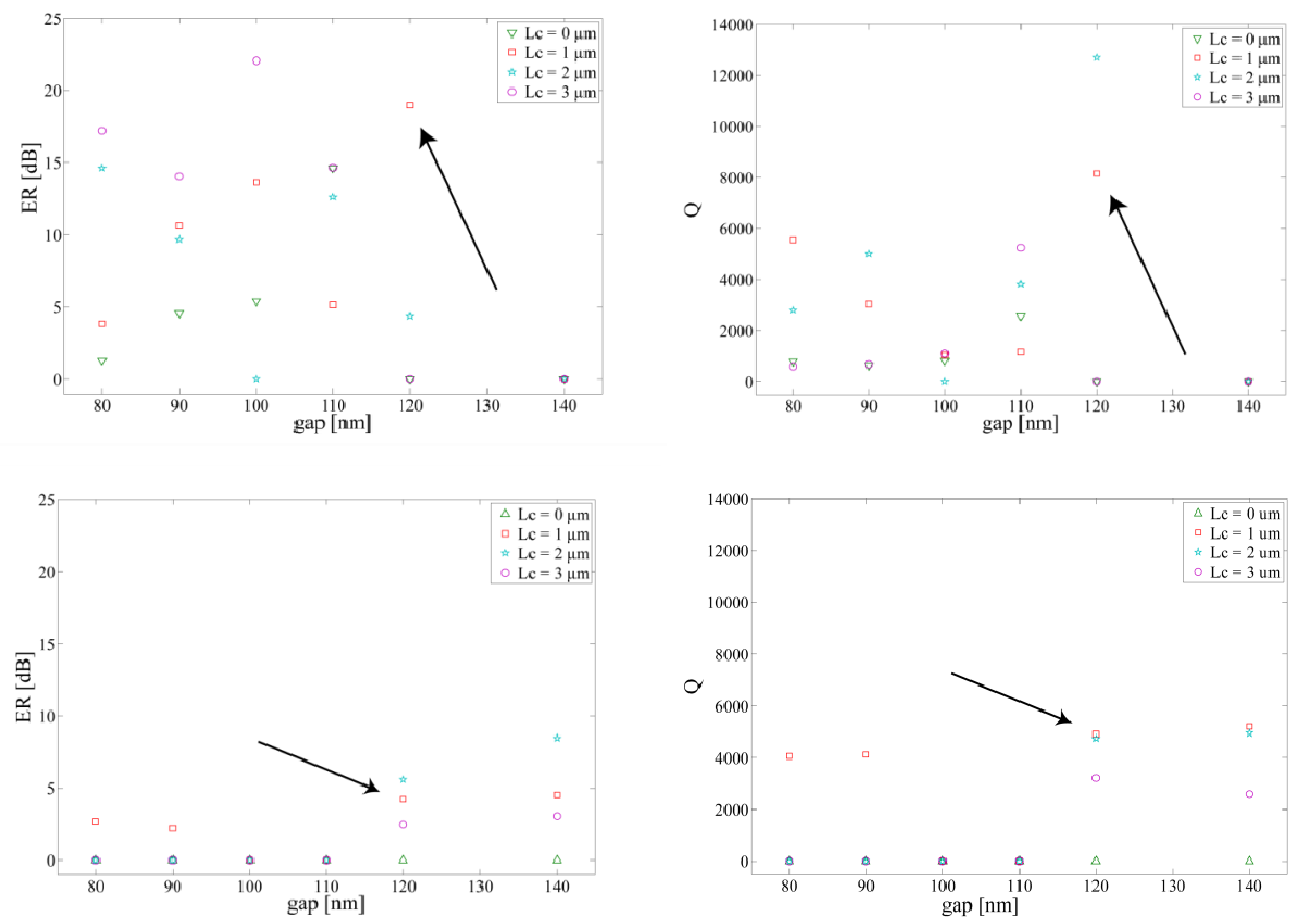

Figure 2: Measured $Q$ (a) and E.R. (b) of a TE mode and $Q$ (c) and E.R. (d) for a TM mode for a microring with water cladding and $a$ width of $520 \mathrm{~nm}$ in function of the gap of the coupling section, for various coupling lengths. The ring which was used for the polymer experiment is indicated. 
Figure 2(a) and 2(b) show the measured (loaded) Q-factor and the extinction ratio (E.R) of a TE mode, while Figure 2(c) and 2(d) show the Q-factor and the extinction ration of a TM mode of a microring resonator with an access waveguide width of $290 \mathrm{~nm}$ and a ring waveguide width of $520 \mathrm{~nm}$, as a function of the gap in the directional coupler. For molecular sensing, a Q factor over 5000 is advisable, while the E.R. should not be less than $5 \mathrm{~dB}$. For the TE mode, we see that for gaps smaller than $120 \mathrm{~nm}$, the E.R. is adequate but the $\mathrm{Q}$ factor drops, indicating that the loss of the TE mode in the ring is too high due to too much coupling in the coupling section. For small gaps it is also not guaranteed that the gap is completely etched, increasing coupling even further. For a gap of $140 \mathrm{~nm}$ (and higher), we see that there are no TE modes at all. For these gaps, the spatial overlap of the electric fields can no longer compensate the phase. On Figure 2(c), we see that there are no appropriate TM modes for gaps smaller than $120 \mathrm{~nm}$. The lower Q-factor of the TM modes in Figure 2(d), compared to those of TE mode in Figure 2(b) is due to the larger water absorption for TM mode. When we combine both workable regions for TE and TM, only rings with a gap of $120 \mathrm{~nm}$ are adequate for sensing. We find that the ring with a coupling length of $1 \mu \mathrm{m}$ performs best. The sizes of the gap as mentioned in Figure 2 are the dimensions as put on the lithographic mask. For small gaps, the printed gaps can be quite different from the ones on the mask. We can conclude that the design window that allows us to excite both a TE and a TM mode simultaneously with one coupling section is relatively small.

\subsection{Extracting the thickness and the refractive index from the wavelength shifts}

The measurement setup allows us to track the change of the wavelength of both the TE and the TM mode of the microring resonator simultaneously. Both depend independently on the thickness $t$ and the refractive index $n$ of the bound layer of polyelectrolytes. In order to extract $t$ and $n$, we require a set of equations that link the wavelength shifts to these independent quantities. Via the equations of Maxwell and perturbation theory, the following set of equations can be found:

$$
\Delta \lambda(n, t)=B \frac{\left(n-n_{b}\right) n f_{p}(t, n)}{1+n^{2} f_{p}(t, n)+n_{b}^{2} f_{b}(t, n)}
$$

Here, $n_{b}$ denotes the bulk refractive index of the cladding fluid while the functions $f_{b}$ and $f_{p}$ and the constant $\mathrm{B}$ can be determined with the help of simulation package Fimmwave. The cladding fluid is the fluid which is present above the polyelectrolyte layer. These functions are highly dependent on the waveguide width $(W)$ and height $(H)$. Since the deviation due to fabrication on both $W$ and $H$ is too high to obtain accurate results, a calibration has to be performed with each sensor prior to the experiment. To obtain the correct width and height, water is flowed over the waveguide and the free spectral range of both modes is measured. Due to a high linearity of both modes in either $W$ or $H$, these can be determined accurately with a $3 \sigma$ error of $96 \mathrm{pm}$ on $W$ and $26 \mathrm{pm}$ on $H$. The mathematical model, the solving procedure and the calibration protocol are described in more detail by Hoste ${ }^{7}$. In the experiment that follows the bulk fluid does not have to be calibrated for, since we use water.

\subsection{Evaluating the influence of noise on the resolution}

Several sources of noise cause an uncertainty on the determination of the wavelength of the resonance mode, translating eventually to a minimal detectable thickness and refractive index of the polyelectrolyte layer. There is noise stemming from the measurement setup itself, such as noise on the wavelength of the laser exciting the microring and shot noise and thermal noise coming from the infrared detector. Secondly there is temperature noise and refractive index noise of the fluidics. There is also a noise contribution coming from fitting the discrete spectrum to a Lorentzian lineshape and extracting the exact position of the resonance.

An estimate of the combined effects of most of these noise factors on the position of the wavelength can be measured experimentally by flowing a fluid at a steady rate over the sensor array, tracking the wavelength of the resonance over time and determining the standard deviation on a linear curve, to correct for time dependent drift. There are however noise factors that are dependent on the analyte and the buffer, such as local variations in concentration, which differ from experiment to experiment. To quantify the noise of our system, we have flowed phosphate buffered saline (PBS) at 30 $\mu \mathrm{l} / \mathrm{min}$ over the sensor array for 11 minutes, gathering 47 resonant wavelengths per mode. We have experimentally obtained this noise as $220 \mathrm{fm}$ for the TM mode and $246 \mathrm{fm}$ for the TE mode. This is in good agreement with the noise determined for a TE mode for a similar microring and lithographic fabrication process ${ }^{9}$. To determine the error in $(t, n)$, the wavelength shifts for a protein layer with $n=1.41-1.45$ RIU and $t=2-9 \mathrm{~nm}$ are simulated, on which Gaussian wavelength noise with $220 \mathrm{fm}$ standard deviation for both modes is superimposed. The average error for a waveguide 
with a width between 450 and $560 \mathrm{~nm}$ is thus determined to be $25-30 \mathrm{pm}$ on thickness and $0.8 \cdot 10^{-3}-1 \cdot 10^{-3}$ on refractive index.

\section{ADSORPTION OF POLYELECTROLYTE LAYERS}

In order to flow the polyelectrolytes over the microrings, microfluidic channels need to be defined on top of the chip. A fluidic cell of $5 \mathrm{~mm}$ high with inlet and outlet is made out of PDMS and bonded to the silicon chip by means of a plasma activation step, followed by a heating step combined with mechanical pressure. The inlet is connected to a pump through a Rheodyne 6-port valve system via tubings that transport the fluids to the chip. This allowed us to switch easily between water, connected to the pump, and a controlled volume of polyelectrolyte dissolved in water, which can be injected in a sample loop connected to the valve. To read out the sensor, a laser is swept over a range of $10 \mathrm{~nm}$ at a speed of $1 \mathrm{~nm} / \mathrm{s}$ with a resolution of $5 \mathrm{pm}$. An optical fiber couples the light to a grating coupler at an angle of 10 degrees with respect to the normal of the plane of the chip. The light propagates through the sensor and is coupled back out with a grating coupler, at which stage it is focused on an infrared camera.

A $2 \mathrm{mg} / \mathrm{ml}$ solution in water is prepared for all the polyelectrolytes: PAH, PSS and PEI. PEI is a cationic hydrophilic polymer which can be used for its adhesive properties, and has recently been used as a surface modification polymer for silicon biosensors ${ }^{12}$. PSS is an anionic polyelectrolyte, while PAH is cationic. By flowing PEI first over the negatively charged silicon surface of the ring resonator, followed by PSS and finally PAH, there should be an electrostatic attraction between the top surface and the polyelectrolyte which is being flowed during all steps. Due to the irregularity of these first few interface layers, the outcome of this experiment can differ from instance to instance. Only in bulk layers, the average layer thickness and refractive index is reproducible ${ }^{8}$. The wavelength shifts of the TE and the TM mode during this experiment are shown in Figure 3.

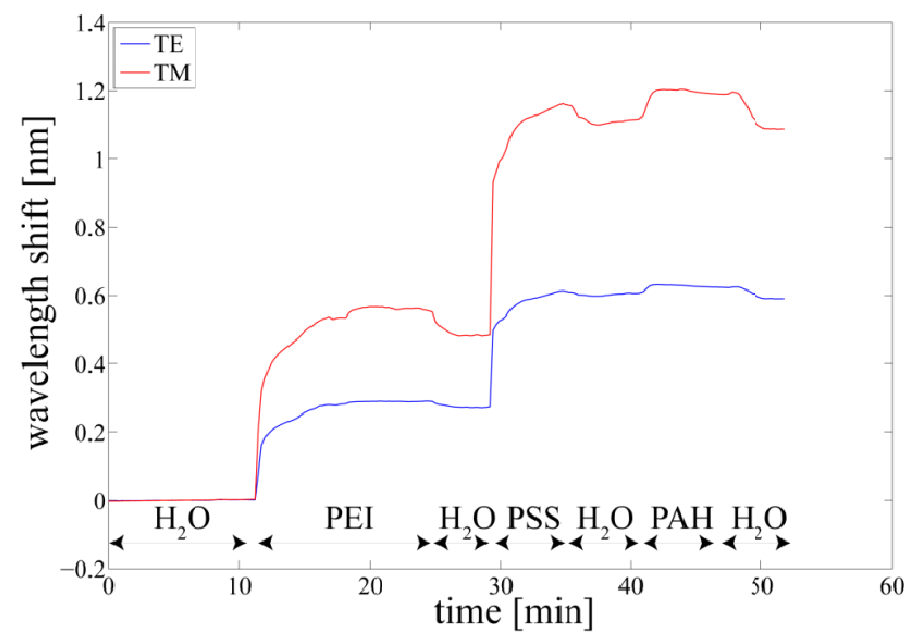

Figure 3: Tracking of the resonant wavelength of both TE and TM modes during the flowing of different polyelectrolytes over the chip surface.

In order to calculate the thickness and refractive index from these shifts, the model as described above is being used. We note that the simulations that provide the data to perform this calculation consist of a $2 \mathrm{D}$ waveguide cross section with a thin layer with unknown thickness and refractive index and a water cladding on top. This means that the computed $(t, n)$ is only correct when water is flown over the chip. Since there is a water stage after every polyelectrolyte stage, this is not a restriction. The mean wavelength shift of the TE mode at the last water stage amounts to $590.7 \mathrm{pm}$ and a standard deviation of $375 \mathrm{fm}$, while the TM mode has a mean shift of $1087.4 \mathrm{pm}$ and a standard deviation of $611 \mathrm{fm}$. The standard deviations computed here are slightly higher than those computed in the section about noise, since we did not correct for linear drift, and the system is molecularly more complex. The calculated thickness and refractive index profile are shown 

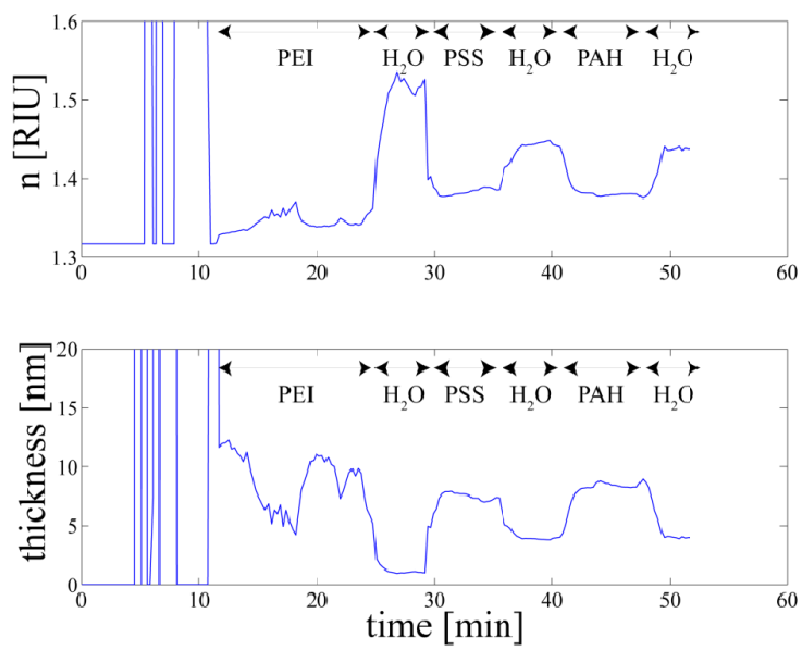

Figure 4: Calculated thickness and refractive index profile of the polymer experiment, as extracted from the shifts of both TE and TM modes, excited in a microring resonator.

in Figure 4. The spikes in the density profile in the first water section are due to the mathematical model: when there is no layer present, the model can solve for any arbitrary thickness and set the refractive index equal to the refractive index of the cladding fluid, or solve for any arbitrary refractive index and set the thickness equal to 0 . The thickness of the complete PEI-PSS-PAH stack amounts to $3.97 \mathrm{~nm}$ with a standard deviation of $53 \mathrm{pm}$, and a refractive index of 1.438 , with a standard deviation of 0.0016 RIU. A thickness of $1.1 \mathrm{~nm}$ per layer is reported for a PEI-PSS-PAH system in dry condition $^{13}$ and a $30 \%$ swelling in water is estimated for PSS-PAH system ${ }^{14}$, such that a thickness of $4.3 \mathrm{~nm}$ is obtained combining both these results. We can conclude that the measured thickness by the dual polarization technique presented here is well in agreement with the reported thickness in literature.

\section{CONCLUSION}

A dual polarization SOI microring has been designed, tailored to resolve thin layers of molecules which bind to the surface of the microring. By tracking the wavelength shift of both a TE and a TM mode that are excited in the microring by using an asymmetrical coupling section, both the thickness and the refractive index of the bound layer can be obtained simultaneously which has been exemplified by flowing a sequence of polyelectrolytes over the sensor which adsorb to the ring surface. A PEI layer, followed by a PSS layer and a PAH layer have bound to the chip and the thickness of this polyelectrolyte system has been calculated to be $3.97 \mathrm{~nm}$ with a standard deviation of $53 \mathrm{pm}$, and the refractive index is calculated to be 1.453 with a standard deviation of 0.0016 RIU. With a resolution below $0.1 \mathrm{~nm}$ in thickness and the capability to solve singular molecular layers in a wet ambience, this sensor has great promises to study molecular binding and conformational change.

\section{REFERENCES}

[1] Connelly, P.R., Vuong, T.M. and Murcko, M.A., "Getting physical to fix pharma," Nat. Chem. 3, 692-695 (2011).

[2] Scannell, J.W., Blanckley, A., Beldon, H. and Warrington, B., "Diagnosing the decline in pharmaceutical R\&D efficiency," Nature Reviews Drug Discovery 11, 191-200 (2012).

[3] Boehr, D.D., Dyson, H.J. and Wright, P.E., "An NMR Perspective on Enzyme Dynamics," Chem. Rev. 106, 3055-3079 (2006).

[4] Some, D. "Light-scattering-based analysis of biomolecular interactions," Biophysical reviews 5(2), 147-158 (2013). 
[5] Melnik, E., Bruck, R., Hainberger, R. and Lämmerhofer M., "Multi-step surface functionalization of polyimide based evanescent wave photonic biosensors and application for DNA hybridization by Mach-Zehnder interferometer," Anal. Chim. Acta 699, 206-215 (2011).

[6] Adam, P., Dostálek, J. and Homola J., "Multiple surface plasmon spectroscopy for study of biomolecular systems," Sensor. Actuat. B-Chem 113( 2), 774-781 (2006).

[7] Hoste, J.-W., Werquin, S., Claes T. and P. Bienstman, "Conformational analysis of proteins with a dual polarisation silicon microring," Opt. Express 22(3), 2807-2820 (2014).

[8] Ladam, G., Schaad, P., Voegel, J.C., Schaaf, P., Decher, G. and Cuisinier, F., "In Situ Determination of the Structural Properties of Initially Deposited Polyelectrolyte Multilayers," Langmuir 31(6), 1249-1255 (2000).

[9] Selvaraja, S.K., Jaenen, P., Bogaerts, W., Van Thourhout, D., Dumon, P. and Baets, R., "Fabrication of Photonic Wire and Crystal Circuits in Silicon-on-Insulator Using 193nm Optical Lithography," J. Lightwave Technol. 0(0), 1-8 (2009).

[10]De Heyn, P., Vermeulen, D., Van Thourhout, D. and Roelkens, G., "Silicon-on-Insulator All-Pass Microring Resonators Using a Polarization Rotating Coupling Section," IEEE Photonic. Tech. L. 24(14), 1176-1178 (2012).

[11] Iqbal, M., Gleeson, M.A., Spaugh, B., Tybor, F., Gunn, W.G., Hochberg, M., Baehr-Jones, T., Bailey, R.C. and Gunn, L.C., "Label-free biosensor arrays based on silicon ring resonators and high-speed optical scanning instrumentation," IEEE J. Quantum Electron. 16(3), 654-661 (2010).

[12] Melnik, E., Muellner, P., Bethge, O., Bertagnolli, E., Hainberger, R. and Laemmerhofer, M., "Streptavidin binding as a model to characterize thiol-ene chemistry-based polyamine surfaces for reversible photonic protein biosensing," Chem. Commun. 50, 2424-2427 (2014).

[13] Kolasińska, M., Krastev, R. and Warszyński, P., "Characteristics of polyelectrolyte multilayers: effect of PEI anchoring layer and posttreatment after deposition," J. Colloid Interf. Sci. 305(1), $46-56$ (2007).

[14] Wong, J.E., Rehfeldt, F., Hanni, P., Tanaka, M. and v. Klitzing, R., "Swelling Behavior of Polyelectrolyte Multilayers in Saturated Water Vapor," Macromolecules 37, 7285-7289 (2004). 